\title{
Neutron diffraction analysis of human manganese superoxide
}

\section{dismutase}

Jahaun Azadmanesh, University of Nebraska Medical Center, Omaha, NE Scott R. Trickel, University of Nebraska Medical Center, Omaha, NE

Kevin L. Weiss, Oak Ridge National Laboratory, Oak Ridge, TN

Leighton Coates, Oak Ridge National Laboratory, Oak Ridge, TN

Gloria E. O. Borgstahl, University of Nebraska Medical Center, Omaha, NE

\begin{abstract}
Superoxide dismutases (SODs) are necessary antioxidant enzymes that protect cells from oxidative stress by converting superoxide, a reactive oxygen species, into molecular oxygen and hydrogen peroxide via cyclic reduction and oxidation at the active site metal. Oxidative stress is involved in many disease states, including cancer, neurological disorders, and heart disease. Despite their protective biological importance, the complete multistep enzymatic mechanisms of SODs are unknown due to limitations in identifying the positions of hydrogen atoms at the active site. Knowledge of the hydrogen positions are critical because (1) they are needed to differentiate the ligands in the active site, which are hypothesized to differ based on the redox state of the active site metal, and (2) they reveal the source and pathway of protons to the catalytic center for proton-assisted electron transfer.
\end{abstract}

The missing structural data on SOD complexes and intermediates can be revealed using Neutron Macromolecular Crystallography (NMC). Neutron diffraction has multiple advantages over X-ray diffraction. (1) Neutrons probe structure without radiation damage, allowing high quality data collection at room temperature. (2) They are inert and do not reduce metals like Xrays do, allowing crystallographic studies on fully oxidized metalloproteins. (3) Hydrogen placement can be identified without the need of $<1 \AA$ data that $X$-ray diffraction requires. Only $\sim 0.5 \%$ of Protein Data Bank (PDB) X-ray crystal structures have high enough resolution to identify hydrogen atoms. Neutron diffraction requires $2.50 \AA$ data or better. The limiting factor for $\mathrm{NMC}$ is the requirement for large crystals and/or deuteration of the sample. Consequently, neutron diffraction will be vital for elucidating the catalytic mechanism of SODs due to their dependence on redox state and exchangeable hydrogens to facilitate catalysis.

Methods are presented on large crystal growth $\left(0.26 \mathrm{~mm}^{3}\right)$ and neutron data collection of human MnSOD with use of perdeuteration and the Macromolecular Neutron Diffractometer beamline at Oak Ridge National Laboratory. The human MnSOD data set provides the largest unit cell edge, $240 \AA$, ever to be collected via neutron diffraction to sufficient resolution, $2.30 \AA$, where hydrogen positions can be observed. 